\title{
Epigenetic modifiers synergize with immune-checkpoint blockade to enhance long-lasting antitumor efficacy
}

\author{
Marina Baretti and Mark Yarchoan \\ The Sidney Kimmel Comprehensive Cancer Center at Johns Hopkins, Baltimore, Maryland, USA
}

\begin{abstract}
Immune-checkpoint inhibitors are firmly established as pillars of cancer therapy, but only a minority of cancer patients currently benefit from these therapies, and therapeutic combinations that can enhance responses are urgently needed. Recently, histone deacetylases (HDACs) have emerged as potential targets for immune modulation, but critical questions remain about their mechanisms of action. In this issue of the $J C I$, Truong et al. assess whether the HDAC inhibitor entinostat can enhance anti-PD-1 treatment in a bladder cancer model. Entinostat promoted a T cellinflamed phenotype and had substantial antitumor efficacy when used in combination with anti-PD-1 therapy. In addition, the authors showed that HDAC inhibition augmented tumor neoantigen presentation, resulting in the immune editing of tumor antigens. This study highlights a mechanism by which epigenetic modifier agents can synergize with immune-checkpoint blockade for enhanced and long-lasting antitumor activity.
\end{abstract}

of patients achieve objective responses, and confirmatory trials of multiple antiPD-1/PD-L1 therapies initially approved in the US through accelerated approval pathways (e.g., atezolizumab, durvalum$\mathrm{ab}$, pembrolizumab) have recently failed to meet their primary end points $(5,6)$. Therapeutic combinations that can synergize with anti-PD-1/PD-L1 therapy will be necessary to increase response rates and improve clinical outcomes.

\section{Epigenetics is a key player in immuno-oncology}

Recent preclinical and clinical data have identified epigenetic deregulation as a critical driver of tumor progression. Epigenetics is the field of study that focuses on the regulation of gene expression in a transmissible but reversible manner, distinguishing epigenetics from the intrinsic DNA sequences that are the focus of genetics. Epigenetics also describes modifications to the DNA sequences, including aberrant DNA methylation patterning, rearrangements in local chromatin structure, posttranslational modifications of histones, and noncoding RNA (7). Histones are organized in nucleosomes whose compaction is regulated by posttranslational modifications occurring on amino acids within the amino terminal and carboxy terminal tails, the most studied being acetylation and methylation. Histone acetylation leads to the euchromatin status, which provides accessibility to transcription factors and gene-expression machinery. Conversely, histone deacetylases (HDACs) make DNA less accessible to transcription factors and consequently repress transcription (8).

HDAC inhibitors (HDACi) can inhibit tumorigenesis and progression by promoting acetylation of histones and nonhistone protein substrates. Two HDACi are FDA approved as monotherapy for the treatment of a rare hematologic malignancy, cutaneous $\mathrm{T}$ cell lymphoma (CTCL), and multiple other HDACi are under clinical 
Immune system evasion

$\downarrow$ Tumor antigen expression

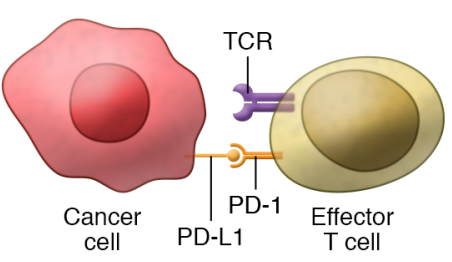

Tumor neoantigen editing

$\uparrow$ Tumor antigen expression

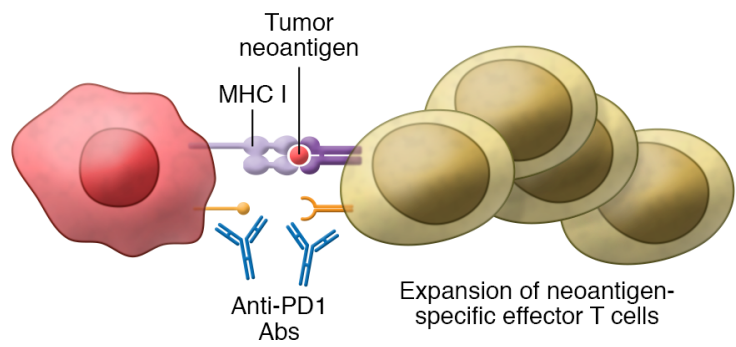

Figure 1. Model for tumor neoantigen editing via epigenetic modifiers. Entinostat promotes tumor cells to express neoantigens. Subsequently, T cells recognize tumor neoantigens, expand, and clear tumor cells. Truong et al. showed that, when combined with anti-PD-1 in bladder cancer models, entinostat enhanced antitumor activity (16).

investigation in different cancer types (9, 10). However, the activity of these agents as monotherapy in a wide range of tumors has been limited (11).

Epigenetic events associated with tumor development and progression regulate ICI expression, tumor-associated antigen presentation, and cancer cell editing by the immune system, and unlike genetic alterations, epigenetic alterations can be reversed pharmacologically in some instances (12).

Until now, studies of the immune implications of HDACi have largely focused on effects of HDACi on specific immune populations and on reversing tumor immunosuppression. Recent work with HDACi has shown the ability of these agents to alter the TME by enhancing the expression of tumor-associated antigens, increasing tumor cell expression of MHC class I and II and increasing the ratio of effector $\mathrm{T}$ cells to immunosuppressive cell types, such as Tregs and myeloid-derived suppressive cells (MDSCs), in multiple different tumor models. Combination therapy of entinostat and anti-PD-1 in animal models suggests that these agents may have complementary or synergistic activity (13-15). However, the precise mechanisms through which HDACi modulate the tumor microenvironment (TME) have been difficult to pin down because HDACi have pleomorphic effects on both tumor cells and immune cells through a wide number of different downstream pathways.

In this issue of the JCI, Truong et al. describe an exciting mechanism through which HDACi therapies can promote antitumor immunity: HDACi can regulate the neoantigen landscape by promoting the transcription of mutation-associated neoantigens and enhancing neoantigenspecific $\mathrm{T}$ cell responses by the host immune system (16).

\section{Cell-autonomous cancer immunoediting effects of epigenetic modifiers}

Truong and colleagues studied the class I-selective HDACi entinostat alone or in combination with anti-PD-1 immunotherapy in preclinical models of bladder cancer (Figure 1). The authors showed that entinostat has antitumor activity in two separate preclinical models of bladder cancer. A series of elegant in vitro experiments characterized the basis for the enhanced antitumor activity observed. The effect was immune dependent and was largely abrogated either in immune-deficient mouse models or through knocking out $\beta_{2}$ microglobulin with CRISPR/Cas9, resulting in MHC class I deficiency. Flow cytometry of immune cells infiltrating the tumors revealed that treatment with entinostat reduced the presence of FoxP3 ${ }^{+}$cells and shifted MDSCs from a monocytic MDSCdominant population to the less immunosuppressive granulocytic MDSCs. Treg and mMDSC depletion due to entinostat treatment was accompanied by increased infiltration by $\mathrm{CD}^{+}$effector memory cells into the TME. The findings support the conclusion that entinostat, rather than being directly cytotoxic to the tumor, has immunomodulatory activity (16).

To address the critical question of how entinostat remodels the TME and promotes an inflamed TME, the authors compared the transcriptional landscape of entinostat- and vehicle-treated tumors. Entinostat treatment enriched immunomodulatory pathways involved in the immune response, including antigen processing and presentation and cytokines/chemokines, thus promoting a $\mathrm{T}$ cell-inflamed phenotype. Genomically, the tumors from entinostat-treated mice uniquely displayed loss of up to 745 expressed neoantigens as compared with those of mice receiving vehicle treatment, which displayed loss of only 15 neoantigens. Genetic evidence of neoantigen depletion was not observed in immunodeficient models, suggesting that immune system-tumor interactions led to tumor immunoediting and subsequent antitumor activity. The authors further demonstrated increased $\mathrm{T}$ cell clonotype sharing and strong $\mathrm{T}$ cell immunoreactivity toward a subset of predicted neoantigens in vitro, suggesting the presence of an active antigen-driven response in these tumors. Entinostat has effects on tumor cells as well as direct effects on lymphocytes. To determine whether entinostat's effects on neoantigen-specific $\mathrm{T}$ cell responses were due to tumor changes or direct effects on the $\mathrm{T}$ cells themselves, the authors performed coculture assays mixing entinostat-pretreated or vehicle-treated $\mathrm{T}$ cells with entinostat-treated or untreated tumors. These experiments convincingly show that entinostat treatment of tumor cells, but not $\mathrm{T}$ cells, was responsible for the increased $\mathrm{T}$ cell killing.

Finally, Truong et al. showed that combination therapy of entinostat with antiPD-1 reduced tumor burden and survival as compared with those of either entinos- 
tat- or anti-PD-1-treated animals. Interestingly, following complete tumor eradication, rechallenge of mice with tumor cell injection did not result in any tumors, suggesting not only that immunoedited tumor clones were eliminated, but also that the mice achieved long-lasting immunological memory. Collectively, these findings indicate the mechanisms by which epigenetic therapy may modulate neoantigens' landscape in tumors, thus providing antigens that can be targeted by the immune system and synergizing with immune-checkpoint blockade for enhanced and long-lasting antitumor activity (16).

\section{Epigenetic agents can overcome resistance to checkpoint inhibitors}

The immune system can either inhibit or favor tumor development and progression. Under pressure from the adaptive immune system, tumor subclones may undergo an editing process that leads to reduced immunogenicity and eventually resistance to immune system recognition and elimination, which begins the escape phase of the cancer immunoediting process (17). We appreciate now that this tumor immune evasion process is due, in part, to reduced expression of neoantigens. Truong et al. elegantly show that epigenetic agents can modulate tumor immunogenicity in a cell-autonomous manner, reestablishing $\mathrm{T}$ cell immunity against neoantigens (16).

We can speculate that similar biological phenomenon of reduced immunogenicity and antigenicity observed here can explain primary and secondary resistance to cancer immunotherapy: the most immunostimulatory antigens are subjected to epigenetic silencing through the immunoselection of less immunogenic disease clones (immune editing) (3). Adding HDAC inhibition to anti-PD-1 treatment against tumors that have developed resistance to checkpoint-blockade immunotherapy may lead the immune system to rerecognize the tumor and downmodulate immune-suppressive elements in the TME, thereby increasing the efficacy of anti-PD-1 therapy. The phase II ENCORE 601 trial assessed the effectiveness of entinostat and pembrolizumab in patients with unresectable or metastatic melanoma that progressed during or following treatment with anti-PD-1 immunotherapy. The study reported nine partial responses and one complete response following the combinatorial treatment, resulting in an objective response rate of $19 \%$ (18). These results suggest that the entinostat/pembrolizumab combination may be an active regimen for patients who never responded to or progressed during treatment with PD-1 inhibitors.

George et al. describe a case of a patient with uterine leiomyosarcoma who experienced near complete remission on anti-PD-1 treatment. Genomic analysis of the treatment-resistant metastasis showed reduced expression of two neoantigens, for which strong immunoreactivity with patient $\mathrm{T}$ cells in vitro was demonstrated, thus suggesting that reduced expression of genes encoding immunogenic neoantigens mediates resistance to immune-checkpoint therapy (19).

A key next step will be identifying a biomarker to better determine which patients will respond to an HDACi combination strategy. Notably, the two bladder cancer model mouse lines used in the Truong et al. study to demonstrate entinostat's effects on tumor immunity have a high number of mutations, whereas entinostat failed to enhance antitumor immunity in a third model of bladder cancer that has a lower TMB (16). This result implies that the positive effects of HDAC on tumor immunity may depend in part on having a high neoantigen burden. This high neoantigen hypothesis is further supported by the clinical experience of HDACi, in which immune-sensitizing activity has been observed in two high tumor types with high TMBs (NSCLC and melanoma), but not in a relatively low TMB tumor type (ovarian cancer) (20). Collectively, these data suggest that entinostat is most successful at making an immune-responsive tumor even more immune responsive, but may not turn an immune-resistant (termed "cold") tumor into an immune-responsive tumor.

However, mutational load represents a challenging biomarker in the clinic. The formation of neoantigens from individual mutations is a stochastic process, and neoepitope quality may be a more important factor than the overall burden of neoantigen quantity in the antitumor immune response. A composite approach of multiple biomarkers will likely be needed, and further research in these areas is ongoing.

\section{Exploiting tumor neoantigens as targets for cancer immunotherapy}

The work by Truong et al. (16) supports the potential of epigenetic modifiers to enhance tumor immunogenicity through modulation of neoantigens' landscape, but associated hurdles and complexities should be considered. First, the optimal dose and sequence of epigenetic drugs should be carefully evaluated. In Truong et al., entinostat provoked antitumor immunity through a cell-autonomous manner. This result may support a sequential strategy with an epigenetic lead-in phase to prime the cancer cell and achieve the desired immunomodulation, followed by combination with ICIs. Doses of less than the maximum that are tolerated might achieve such effects. Second, the epigenetic agent's therapeutic window relies on identifying pharmacodynamic biomarkers to direct patient care and exploring a strong rationale for combining epigenetic-immune therapy in appropriate preclinical models. Well-designed clinical trials are needed, as the mechanisms by which epigenetic modulators elicit immunomodulatory effects can be tumor specific and different in immune-naive patients as compared with immune-resistant ones (21).

Cancer is both a genetic and an epigenetic disease (7); enhancing our comprehension about the precise relationships among epigenetic aberrations, the immune system, and the consequences for cancer cell phenotypes could have tremendously important translational implications.

Address correspondence to: Mark Yarchoan or Marina Baretti, 1650 Orleans St., Baltimore, Maryland 21287, USA. Phone: 410.955.2957; Email: mark.yarchoan@ jhmi.edu (MY). Phone: 410.502.9454; Email: mbarett1@jh.edu (MB).

1. Pardoll DM. The blockade of immune checkpoints in cancer immunotherapy. Nat Rev Cancer. 2012;12(4):252-264.

2. Yarchoan M, et al. Tumor mutational burden and response rate to PD-1 inhibition. $N$ Engl JMed. 2017;377(25):2500-2501.

3. Yarchoan M, et al. Targeting neoantigens to augment antitumour immunity. Nat Rev Cancer. 2017;17(4):209-222.

4. Haslam A, Prasad V. Estimation of the percentage of us patients with cancer who are eligible for and respond to checkpoint inhibitor immunotherapy drugs. JAMA Netw Open. 
2019;2(5):e192535.

5. Beaver JA, Pazdur R. "Dangling” accelerated approvals in oncology. $N$ Engl JMed. 2021;384(18):e68.

6. Chen H-L, et al. Immune checkpoints inhibitors and chemotherapy as first-line treatment for metastatic urothelial carcinoma: a network meta-analysis of randomized phase III clinical trials. Cancers (Basel). 2021;13(6):1484.

7. Jones PA, Baylin SB. The epigenomics of cancer. Cell. 2007;128(4):683-692.

8. Tough DF, et al. Epigenetic drug discovery: breaking through the immune barrier. Nat Rev Drug Discov. 2016;15(12):835-853.

9. Whittaker SJ, et al. Final results from a multicenter, international, pivotal study of romidepsin in refractory cutaneous T-cell lymphoma. JClin Oncol. 2010;28(29):4485-4491.

10. Olsen EA, et al. Phase IIB multicenter trial of vorinostat in patients with persistent, progressive, or treatment refractory cutaneous T-cell lymphoma. J Clin Oncol. 2007;25(21):3109-3115.

11. Khan O, Thangue NBL. HDAC inhibitors in cancer biology: emerging mechanisms and clinical applications. Immunol Cell Biol. 2012;90(1):85-94.

12. Cycon KA, et al. Histone deacetylase inhibitors activate CIITA and MHC class II antigen expression in diffuse large B-cell lymphoma. Immunology. 2013;140(2):259-272.

13. Kim K, et al. Eradication of metastatic mouse cancers resistant to immune checkpoint blockade by suppression of myeloid-derived cells. Proc Natl Acad Sci U S A. 2014;111(32):11774-11779.

14. Orillion A, et al. Entinostat neutralizes myeloid-derived suppressor cells and enhances the antitumor effect of PD-1 inhibition in murine models of lung and renal cell carcinoma. Clin Cancer Res. 2017;23(17):5187-5201.

15. Christmas BJ, et al. Entinostat converts immune-resistant breast and pancreatic cancers into checkpoint-responsive tumors by reprogramming tumor-infiltrating MDSCs. Cancer Immunol Res. 2018;6(12):1561-1577.

16. Truong AS, et al. Entinostat induces antitumor immune responses through immune editing of tumor neoantigens. J Clin Invest. 2021;131(16):e138560

17. O'Donnell JS, et al. Cancer immunoediting and resistance to T cell-based immunotherapy. Nat Rev Clin Oncol. 2019;16(3):151-167.

18. Sullivan RJ, et al. Abstract CT072: efficacy and safety of entinostat (ENT) and pembrolizumab (PEMBRO) in patients with melanoma previously treated with anti-PD1 therapy. Paper presented at: AACR Annual Meeting; March 29-April 3, 2019; Atlanta, Georgia, USA. https://cancerres. aacrjournals.org/content/79/13_Supplement/ CT072. Accessed July 22, 2021.

19. George S, et al. Loss of PTEN is associated with resistance to Anti-PD-1 checkpoint blockade therapy in metastatic uterine leiomyosarcoma. Immunity. 2017;46(2):197-204.

20. Hellmann MD, et al. Entinostat plus pembrolizumab in patients with metastatic NSCLC previously treated with Anti-PD-(L)1 therapy. Clin Cancer Res. 2021;27(4):1019-1028.

21. Aspeslagh S, et al. Epigenetic modifiers as new immunomodulatory therapies in solid tumours. Ann Oncol. 2018;29(4):812-824. 